\title{
Seminare 2012
}

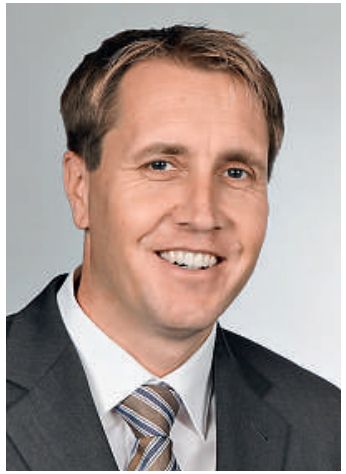

René Häller, Geschäftsführer FMH Consulting Services

\section{Praxiseröffnung/-übernahme}

Das Seminar richtet sich an Ärztinnen und Ärzte, welche vor einer Praxiseröffnung (Einzel-/Gruppenpraxis), dem Einstieg in eine Gruppenpraxis oder vor einer Praxisübernahme stehen.

\section{Themen}

Juristische Aspekte (Praxisbewilligung, Zulassung zur Sozialversicherung, Vertragswesen), Gesellschaftsformen / Ehe- und Erbrecht (Trennung Privat- vom Geschäftsvermögen, Ehegüterstand, Erbschaftsplanung), Praxiseinrichtung (Inneneinrichtung, Kostenberechnung), Praxisadministration (Leistungserfassungs- und Abrechnungssysteme), Unternehmensbewertung einer Arztpraxis (Berechnung und Beurteilung des Unternehmenswertes), Finanzierung der Arztpraxis (Businessplan, Kredite, Absicherungsmöglichkeiten), Versicherungen/ Vorsorge/Vermögen (Personen- und Sachversicherungen, Vorsorgeplanung).

\section{Kosten/Sponsoren}

Für FMH Services-Mitglieder kostenlos. Die Kosten werden durch diverse Sponsoren (siehe www.fmhservices.ch) gedeckt.

\section{Daten}

\begin{tabular}{|c|c|c|}
\hline K01 & $\begin{array}{l}\text { Donnerstag, } \\
\text { 8. März } 2012 \\
9.00-16.30 \text { Uhr }\end{array}$ & $\begin{array}{l}\text { Zürich } \\
\text { FMT }\end{array}$ \\
\hline K02 & $\begin{array}{l}\text { Donnerstag, } \\
\text { 3. Mai } 2012 \\
\text { 16.00-20.30 Uhr }\end{array}$ & $\begin{array}{l}\text { St. Gallen } \\
\text { Hotel Radisson }\end{array}$ \\
\hline
\end{tabular}

Obwohl rund 40\% aller Ärztinnen und Ärzte früher oder später eine eigene Arzt-/ Gruppenpraxis führen und nebst dem medizinischen Know-how auch jenes eines Unternehmers innehaben sollten, wird im Verlauf des Medizinstudiums die Thematik der Unternehmensführung nie oder nur kaum fokussiert. Die FMH Services, welche sich um die betriebswirtschaftlichen Angelegenheiten ihrer Genossenschafter kümmern, schliessen diese Lücke und bieten dazu spezifische Seminare an. In diesen Seminaren wer- den den Ärztinnen und Ärzten Grundlagen vermittelt für den Einstieg in die eigene Arzt-/Gruppenpraxis, deren Führung und deren optimale Übergabe an einen/eine Nachfolger/in. Die Ärztinnen und Ärzte werden auf den Umgang und die Zusammenarbeit mit Behörden sowie Fachspezialisten, wie Treuhänder, Versicherungs- und Finanzberater, Kreditberater bei Banken, Juristen, Architekten usw., vorbereitet. Die einzelnen Themen werden von ausgewiesenen Experten der FMH Services referiert.

\begin{tabular}{|c|c|c|}
\hline 03 & $\begin{array}{l}\text { Donnerstag, } \\
\text { 14. Juni } 2012 \\
\text { 9.00-16.30 Uhr }\end{array}$ & $\begin{array}{l}\text { Bern } \\
\text { Schmiedstube }\end{array}$ \\
\hline K04 & $\begin{array}{l}\text { Donnerstag, } \\
\text { 6. September } 2012 \\
9.00-16.30 \text { Uhr }\end{array}$ & $\begin{array}{l}\text { Zürich } \\
\text { FMT }\end{array}$ \\
\hline 40 & $\begin{array}{l}\text { Donnerstag, } \\
\text { 8. November } 2012 \\
9.00-16.30 \text { Uhr }\end{array}$ & $\begin{array}{l}\text { Basel } \\
\text { Hotel Victoria }\end{array}$ \\
\hline
\end{tabular}

\section{Praxisuibergabe}

Das Seminar richtet sich an zukünftige Praxisübergeber/innen. Hinweis: Vor allem aus steuer- und vorsorgeplanerischer Sicht lohnt es sich, sich bereits frühzeitig (5-10 Jahre) mit diesem Thema auseinanderzusetzen.

\section{Themen}

Juristische Aspekte (Praxisübergabevertrag, allg. Vertragswesen, Übergabe der Krankengeschichten), Unternehmensbewertung einer Arztpraxis (Berechnung Inventarwert und Goodwill als Verhandlungsbasis), Versicherungen/Vorsorge/Vermögen (Übergabe/Auflösung von Versicherungsverträgen, Pensions- und Finanzplanung), Steuern (Steueraspekte bei der Praxisübergabe, Optimierung der steuerlichen Auswirkungen, Liquidations- und Grundstückgewinnsteuer, Bestimmung des optimalen Übergabezeitpunktes).

\section{Kosten/Sponsoren}

Für FMH Services-Mitglieder kostenlos. Die Kosten werden durch diverse Sponsoren (siehe www.fmhservices.ch) gedeckt.

\section{Daten}

\begin{tabular}{|c|c|c|}
\hline K06 & $\begin{array}{l}\text { Donnerstag, } \\
\text { 15. März } 2012 \\
\text { 13.30-18.00 Uhr }\end{array}$ & $\begin{array}{l}\text { Zürich } \\
\text { FMT }\end{array}$ \\
\hline K07 & $\begin{array}{l}\text { Donnerstag, } \\
\text { 10. Mai } 2012 \\
\text { 16.00-20.30 Uhr }\end{array}$ & $\begin{array}{l}\text { St. Gallen } \\
\text { Hotel Radisson }\end{array}$ \\
\hline K08 & $\begin{array}{l}\text { Donnerstag, } \\
\text { 21. Juni } 2012 \\
\text { 13.30-18.00 Uhr }\end{array}$ & $\begin{array}{l}\text { Bern } \\
\text { Schmiedstube }\end{array}$ \\
\hline K09 & $\begin{array}{l}\text { Donnerstag, } \\
\text { 13. September } 2012 \\
\text { 13.30-18.00 Uhr }\end{array}$ & $\begin{array}{l}\text { Zürich } \\
\text { FMT }\end{array}$ \\
\hline K10 & $\begin{array}{l}\text { Donnerstag, } \\
\text { 15. November } 2012 \\
\text { 13.30-18.00 Uhr }\end{array}$ & $\begin{array}{l}\text { Basel } \\
\text { Hotel Victoria }\end{array}$ \\
\hline
\end{tabular}

\section{Finanz- und Steuerplanung}

Das Seminar richtet sich an Praxiseröffner/ innen, Praxisübernehmer/innen sowie an bereits praxistätige Ärztinnen und Ärzte.

\section{Themen}

Finanzplanung (Businessplan, Buchhalterische Massnahmen vor Praxiseröffnung/ -übernahme, Standardkontenplan für Ärzte, System der doppelten Buchhaltung, EDVunterstützte Buchführungslösung), Steuern (Steueraspekte bei Eintritt in die Selbständigkeit, Steuerfallen und Steuerrisiken, optimierte Steuerplanung).

\section{Kosten/Sponsoren}

Für FMH Services-Mitglieder kostenlos. Die Kosten werden durch diverse Sponsoren (siehe www.fmhservices.ch) gedeckt. 


\section{Daten}

$\begin{array}{ll}\text { K11 } & \begin{array}{l}\text { Donnerstag, } \\ \text { 22. März 2012 }\end{array} \\ \begin{array}{l}\text { 13.30-18.00 Uhr } \\ \text { K12 }\end{array} & \begin{array}{l}\text { Zürich } \\ \text { Donnerstag, }\end{array} \\ \begin{array}{l}\text { 20. September 2012 } \\ \text { 13.30-18.00 Uhr }\end{array} & \begin{array}{l}\text { Bern } \\ \text { Schmiedstube }\end{array}\end{array}$

\section{Praxiscomputerworkshop}

Der Workshop richtet sich an praxiseröffnende sowie an bereits praxistätige Ärztinnen und Ärzte.

\section{Inhalt}

Der Workshop beinhaltet eine Einführung in die Anforderungen an ein Praxisinformationssystem sowie Unterstützung im Evaluationsprozess. Anhand einer modernen vernetzten Praxisinfrastruktur werden die Beurteilungskriterien für eine praxis- und zukunftstaugliche Softwarelösung dargestellt. Die Workshopteilnehmer/innen erhalten die einmalige Möglichkeit, sechs führende Praxisinformationssysteme (Leistungserfassung, elektronisches Abrechnen unter Einbezug der TrustCenter, Agendaführung, Statistiken, Laborgeräteeinbindung, elektronische Krankengeschichte, Finanzbuchhaltungslösungen usw.) kennenzulernen.

\section{Ziel}

Die Teilnehmer/innen erhalten einen Anforderungskatalog, welcher ihnen erlaubt, ihre Vorstellungen für ein modernes Praxisinformationssystem besser $\mathrm{zu}$ formulieren und diese dem Softwarehersteller zu dessen Vorbereitung $\mathrm{zu}$ kommunizieren. Zudem erhalten sie einen ersten Überblick über führende Softwarelösungen.

\section{Kosten}

Für FMH Services-Mitglieder kostenlos.

\section{Daten}

\begin{tabular}{|c|c|c|}
\hline K13 & $\begin{array}{l}\text { Donnerstag, } \\
\text { 29. März } 2012 \\
\text { 13.30-18.00 Uhr }\end{array}$ & $\begin{array}{l}\text { Zürich } \\
\text { Technopark }\end{array}$ \\
\hline K14 & $\begin{array}{l}\text { Donnerstag, } \\
\text { 28. Juni } 2012 \\
\text { 13.30-18.00 Uhr }\end{array}$ & $\begin{array}{l}\text { Bern } \\
\text { BERNEXPO }\end{array}$ \\
\hline K15 & $\begin{array}{l}\text { Donnerstag, } \\
\text { 29. November } 2012 \\
\text { 13.30-18.00 Uhr }\end{array}$ & $\begin{array}{l}\text { Olten } \\
\text { Stadttheater }\end{array}$ \\
\hline
\end{tabular}

\section{Röntgen in der Arztpraxis}

Das Seminar richtet sich an Ärztinnen und Ärzte mit bestehender Praxis und an solche, die vor einer Praxiseröffnung oder Praxis- übernahme stehen. Das Seminar ist auf maximal 15 Teilnehmer/innen beschränkt.

\section{Themen}

Vom konventionellen zum digitalen Röntgen, Rentabilität Röntgen in der Arztpraxis, Neue Vorschriften, Evaluation und Beschaffung neuer oder gebrauchter Anlagen, Komplette Marktübersicht mit Preisen und Leistungskomponenten. Die Seminarteilnehmer erstellen und bearbeiten innerhalb des Seminars digitale Röntgenbilder und erhalten eine komplexe Dokumentation über alle Themen - ein Vademekum rund ums Röntgen.

\section{Kosten}

300 CHF (inkl. sämtlicher Kursunterlagen und Verpflegung).

\section{Datum}

$\begin{array}{ll}\text { K16 Donnerstag, } & \\ \text { 23. August } 2012 & \text { Niederscherli } \\ 09.30-16.00 \mathrm{Uhr} & \text { WIROMA AG }\end{array}$

\section{Jahre vor Pensionierung}

Das Seminar richtet sich an Ärztinnen und Ärzte, welche die Pensionierung (auch eine frühzeitige) nicht dem Zufall überlassen wollen.

\section{Inhalt}

Reich in Rente? Behandlung von Fragen zu einem möglichen Kapitalbedarf, zu möglichen Vorsorgelösungen sowie zum steuerlich optimierten Vorgehen. Treuhand und Altersvorsorge? Aufzeigen von Möglichkeiten, wie ein Praxisverkauf sowie eine ideale Altersplanung aufeinander abgestimmt werden kann und wie dies in vielen Fällen zu massiven Steuereinsparungen führen kann. Planung der Altersvorsorge! Für die Planung der Altersvorsorge kann der zukünftige Bedarf aufgrund der Planungsmöglichkeiten genau anvisiert werden, und die zukünftigen Einkünfte können in Bezug auf Höhe, Steuern und Sicherheit optimal geplant werden.

\section{Kosten}

100 CHF (inkl. sämtlicher Kursunterlagen und Verpflegung).

\section{Daten}

\begin{tabular}{|lll}
\hline K30 & $\begin{array}{l}\text { Donnerstag, } \\
\text { 24. Mai 2012 } \\
\text { 18.00-20.30 Uhr }\end{array}$ & $\begin{array}{l}\text { Aarau } \\
\text { Gasthaus zum } \\
\text { Schützen }\end{array}$ \\
\hline K31 & $\begin{array}{l}\text { Donnerstag, } \\
\text { 27. September 2012 } \\
\text { 18.00-20.30 Uhr }\end{array}$ & Luzern \\
\hline
\end{tabular}

\section{Tarifwerk TARMED - Einführungskurs}

Das Seminar richtet sich an Ärztinnen und Ärzte mit bestehender Praxis und an solche, die kurz vor einer Praxiseröffnung oder Praxisübernahme stehen. Der Einführungskurs vermittelt den Kursteilnehmern die Grundlagen des Tarifwerkes TARMED. Die Seminarteilnehmerzahl ist begrenzt.

\section{Themen}

Fakten (Gesetzliche und vertragliche Grundlagen), Struktur (Tarifbrowser, Grundstruktur des Tarifwerkes, Regelhierarchie, Leistungsblöcke, Leistungsgruppen), Generelle Interpretationen (Wichtigste generelle Interpretationen), Parameter einer Tarifposition (Alle Parameter einer Tarifposition), Tarifpositionen aus dem Kapitel 00 Grundleistungen (Diverse Tarifpositionen aus dem Kapitel 00 Grundleistungen), Praxislabor und Präsenzdiagnostik (Neue Analyseliste), Organisationen und Informationsquellen (www.tarmedsuisse.ch usw).

Kosten

200 CHF (inkl. Kursunterlagen).

\section{Daten}

\begin{tabular}{|c|c|c|}
\hline K60 & $\begin{array}{l}\text { Dienstag, } \\
\text { 20. März } 2012 \\
\text { 14.00-17.15 Uhr }\end{array}$ & $\begin{array}{l}\text { Olten } \\
\text { Hotel Arte }\end{array}$ \\
\hline K61 & $\begin{array}{l}\text { Dienstag, } \\
\text { 22. Mai } 2012 \\
\text { 14.00-17.15 Uhr }\end{array}$ & $\begin{array}{l}\text { Olten } \\
\text { Hotel Arte }\end{array}$ \\
\hline & $\begin{array}{l}\text { Dienstag, } \\
\text { 18. September } 2012 \\
\text { 14.00-17.15 Uhr }\end{array}$ & $\begin{array}{l}\text { Olten } \\
\text { Hotel Arte }\end{array}$ \\
\hline
\end{tabular}

\section{Praxismarketing für Ärzte}

Das Seminar richtet sich an Ärztinnen und Ärzte, welche vor einer Praxiseröffnung stehen oder bereits eine Praxis führen.

\section{Themen}

Der Erfolg einer Arztpraxis ist unlösbar mit gutem Service und Kundenorientierung verbunden. Damit sind mehr als gute Umgangsformen gemeint. Echter Service geht tiefer und bietet den Patientinnen und Patienten - oder Kunden - einen direkten Nutzen. Aus einer Arztpraxis wird DIE Praxis.

Die Herausforderung ist nicht, Service für Patienten zu leisten, sondern Service für Patienten aus der Sicht des Patienten erlebbar zu machen. Dazu gehört, dass man seine Kunden versteht, auf ihre Bedürfnisse vorbereitet ist, sie um Informationen bittet, ihnen genau zuhört, Verantwortung für das eigene Tun übernimmt, über das normale 
Mass hinaus engagiert zu sein, Menschen zu überraschen, und das immer regelmässig und auf gleichbleibend hohem Niveau!

Das Wichtigste zu den Themen Telefon, Empfang, Teamentwicklung wird diskutiert, die wichtigsten Werbemöglichkeiten besprochen. Nicht das Aussergewöhnliche wollen wir tun, sondern das Gewöhnliche aussergewöhnlich gut!

\section{Kosten}

300 CHF (inkl. sämtlicher Kursunterlagen und Verpflegungen).

\section{Daten}

K63 Mittwoch,

14. März 2012 09.00-16.30 Uhr

Zürich FMT

K64 Mittwoch 12. September 2012 09.00-16.30 Uhr

Bern Schmiedstube

\section{Telefonseminar für MPA}

\section{(bzw. Praxisteam)}

Das Seminar richtet sich an MPAs, welche im telefonischen Kundenkontakt stehen bzw. Auszubildende, welche im professionellen Telefonieren angeleitet werden sollen. Die «Visitenkarte» der Arztpraxis soll den modernen Anforderungen gerecht werden.

\section{Themen}

Die Medizinische Praxisassistentin ist die Visitenkarte der Praxis. Sie hat den ersten und den letzten Kontakt mit den Patienten und beeinflusst wesentlich das Image der Arztpraxis. MPAs repräsentieren die Unternehmenskultur, organisieren die Praxis und sind somit ein wesentlicher Bestandteil für den Unternehmenserfolg. «You never get a second chance to make a first impression.»

Der erste Eindruck wird durch den ersten Kontakt geprägt! Und fast immer findet der erste Kontakt, den ein Patient zu seiner Arztpraxis aufnimmt, über das Telefon statt.

Das richtige Telefonieren, das gerade in der Arztpraxis von grosser Bedeutung ist, wird einem nicht in die Wiege gelegt, sondern muss erlernt werden.

\section{Kosten}

300 CHF (inkl. sämtlicher Kursunterlagen und Verpflegungen).

Praxisteams erhalten einen Rabatt von $20 \%$ pro Teilnehmer/in.

\section{Daten}

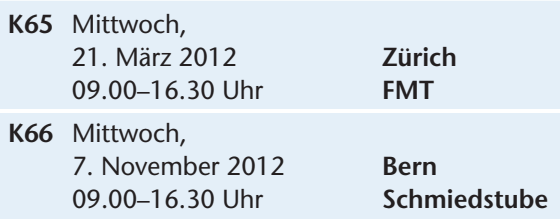

\section{Gruppenpraxis}

Der Grossteil der in Ausbildung stehenden Ärztinnen und Ärzte kann sich eine freipraktizierende Tätigkeit nur in einer Gruppenpraxis vorstellen. Für Einzelpraxen in ländlichen wie auch bereits in städtischen Gebieten wird es immer schwieriger, eine Nachfolgerin oder einen Nachfolger zu finden. Die Neugründung einer Gruppenpraxis oder der Zusammenschluss von bestehenden Einzelpraxen erhöht die Chance einer langfristig erfolgreichen Praxisführung und erleichtert die Nachfolgeplanung erheblich. Nebst den persönlichen Motiven für eine Gruppenpraxis ist es unumgänglich, sich mit den betriebswirtschaftlichen Fragestellungen rechtzeitig auseinanderzusetzen.

\section{Themen}

Unternehmerische Gedanken: Die Unterschiede der Einzel- und Gruppenpraxis sowie deren Führung (Unternehmen, Mitarbeiter) werden aufgezeigt. Welches sind die Anforderungen an das Personal und wie wird die Planung einer Gruppenpraxis projektbezogen organisiert?

Praxismodelle / Rechtsformen / Finanzen / Steuern: Die einzelnen Praxismodelle und die möglichen Rechtsformen (Einzelpraxis, Junior-Senior-Partner-System, Infrastruktur AG / GmbH, AG / GmbH Lösung mit Vollintegration) werden transparent aufgezeigt. Bezüglich Finanzen und Steuern werden die unterschiedlichen Finanzierungsarten erklärt, die Fragestellung betreffend Bewirtschaftung und Geldmitteleinsatz beantwortet und die strategische und operative Steuerplanung (inkl. Mehrwertsteuerproblematik in der Gruppenpraxis) aufskizziert.

Versicherung / Vorsorge: Welches sind die wesentlichen Unterschiede in der Vorsorgesituation bei einer juristischen Person (AG, GmbH) im Vergleich zur Einzelfirma? Welches sind die unterschiedlichen persönlichen Bedürfnisse (z.B. Arzt mit/ohne Familie) hinsichtlich versicherungs- und vorsorgetechnischer Abdeckung in der Gruppenpraxis? Was gilt es bezüglich Berufshaftpflicht zu beachten (wer muss wann was abschliessen) und wo liegen die steuerlichen Konse- quenzen bei einer juristischen Person und der privaten Vorsorge?

Praxisbericht: Der Erfahrungsbericht eines Arztes (Mitgründer einer Gruppenpraxis) aus der Region über die Gründungsphase, die Schwierigkeiten (wo, wann ist externe Unterstützung sinnvoll?), die Organisation und die Führungsprozesse einer Gruppenpraxis rundet das Seminar ab.

\section{Kosten/Sponsoren}

Für FMH Services-Mitglieder kostenlos. Die Kosten werden durch diverse Sponsoren (siehe www.fmhservices.ch) gedeckt.

\section{Daten}

\begin{tabular}{|lll|}
\hline K80 & $\begin{array}{l}\text { Donnerstag, } \\
\text { 31. Mai 2012 } \\
\text { 13.30-18.00 Uhr }\end{array}$ & $\begin{array}{l}\text { Luzern } \\
\text { Hotel } \\
\text { Continental }\end{array}$ \\
\hline K81 & $\begin{array}{l}\text { Donnerstag, } \\
\text { 22. November 2012 } \\
\text { 13.30-18.00 Uhr }\end{array}$ & $\begin{array}{l}\text { Aarau } \\
\text { Gasthaus }\end{array}$ \\
\hline
\end{tabular}

\section{Crashkurs Versicherungsmedizin}

Das Seminar richtet sich an Ärztinnen und Ärzte, vom Assistenzarzt bis zum Klinikdirektor, vom Grundversorger bis zum Superspezialisten. Das Seminar wird in $\mathrm{Zu}$ sammenarbeit mit der Schweiz. Gesellschaft der Vertrauens- und Versicherungsärzte (SGV) durchgeführt.

\section{Themen}

Durch Fallbeispiele lernen die Teilnehmenden die Spielregeln der Versicherungsmedizin von KVG, UVG und IVG kennen und wissen danach, welche Hürden durch Gesetze und Verordnungen entstanden sind. Am Schluss des Kurses ist allen bekannt, in welchen Gesetzen die wesentlichen Aspekte der Versicherungsmedizin behandelt werden, welche Ämter dafür zuständig sind und wo genau diese Regeln und Verordnungen nachgelesen werden können.

\section{Kosten}

100 CHF (inkl. sämtlicher Kursunterlagen und Verpflegungen).

\section{Daten \\ K90 Donnerstag, 26. April 2012 13.00-16.00 Uhr Zürich FMT \\ K91 Donnerstag, 23. August 2012 Bern 13.30-16.00 Uhr Schmiedstube}

\section{Anmeldung und Auskunft}

www.fmhservices.ch oder FMH Consulting 
Services, Cornelia Steinmann, Burghöhe 1, 6208 Oberkirch, Tel. 04192500 77, Fax 041 9210586.

\section{Hinweis}

Bei sämtlichen Seminaren, bei denen die Kosten teilweise oder gänzlich von Seminar- sponsoren gedeckt werden, werden die Teilnehmeradressen den jeweiligen Sponsoren zur Verfügung gestellt.

\section{Annullierungsbedingungen}

Bei Abmeldungen oder Fernbleiben werden folgende Unkostenbeiträge erhoben:

\section{Séminaires / Seminari 2012}

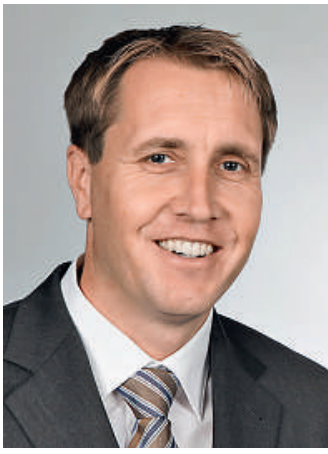

René Häller, Directeur FMH Consulting Services

Bien qu'environ $40 \%$ de tous les médecins ouvrent tôt ou tard leur propre cabinet médical et ont besoin de connaissances en gestion d'entreprise hormis leur savoir-faire médical, cette thématique n'est souvent pas ou peu abordée. FMH Services qui s'occupe de la gestion d'entreprise de ses membres, comble désormais cette lacune en proposant des séminaires spécifiques. Dans ces séminaires, les médecins peuvent acquérir les bases nécessaires pour ouvrir leur propre cabinet médical ou un cabinet de groupe ou pour remettre leur cabinet de manière optimale. Les médecins obtiennent également des conseils sur la manière de collaborer avec les autorités ou avec des spécialistes tels que des experts-comptables, des conseillers financiers, en assurance ou en crédits bancaires, des juristes, des architectes, etc. Ces différents thèmes sont abordés par des experts qualifiés de FMH Services.

\section{Ouverture et reprise d'un cabinet médical}

Le séminaire est destiné aux médecins sur le point d'ouvrir ou de reprendre un cabinet médical.

\begin{abstract}
Contenu
Business plan (préparation du plan de financement et crédit d'exploitation, financement par la banque), Aménagement (implantation, projet et concept d'aménagement, choix du mobilier, budget), Estimation d'un cabinet (inventaire et goodwill), Administration d'un cabinet médical (dans le cabinet, par la banque), Assurances (toutes les assurances à l'intérieur et autour du cabinet), Passage du statut de salarié à celui d'indépendant et fiscalité.
\end{abstract}

\section{Frais/Sponsors}

Gratuit pour les membres FMH Services. Les coûts sont pris en charge par divers sponsors (voir www.fmhservices.ch).

\section{Dates}

\begin{tabular}{|c|c|c|}
\hline K20 & $\begin{array}{l}\text { Jeudi } \\
8 \text { mars } 2012 \\
13.30-18.00 \mathrm{~h}\end{array}$ & $\begin{array}{l}\text { Lausanne } \\
\text { World } \\
\text { Trade Center }\end{array}$ \\
\hline K21 & $\begin{array}{l}\text { Jeudi } \\
31 \text { mai } 2012 \\
13.30-18.00 \mathrm{~h}\end{array}$ & $\begin{array}{l}\text { Genève } \\
\text { Crowne Plaza }\end{array}$ \\
\hline K22 & $\begin{array}{l}\text { Jeudi } \\
13 \text { septembre } 2012 \\
17.00-21.30 \mathrm{~h}\end{array}$ & $\begin{array}{l}\text { Lausanne } \\
\text { World } \\
\text { Trade Center }\end{array}$ \\
\hline K23 & $\begin{array}{l}\text { Jeudi } \\
22 \text { novembre } 2012 \\
17.00-21.30 \mathrm{~h}\end{array}$ & $\begin{array}{l}\text { Genève } \\
\text { Crowne Plaza }\end{array}$ \\
\hline
\end{tabular}

\section{Remise d'un cabinet médical}

Le séminaire s'adresse aux médecins désirant remettre un cabinet médical.

\section{Contenu}

Aspects juridiques (autour du contrat de remise/reprise), Estimation d'inventaire et goodwill d'un cabinet, Assurances (prévoyance, assurances à l'intérieur et autour
- 50 CHF pro Person ab 14 Tage vor Seminarbeginn;

- 100 CHF pro Person ab 7 Tage vor Seminarbeginn oder Fernbleiben.

du cabinet), Conséquences fiscales d'une remise.

\section{Frais/Sponsors}

Gratuit pour les membres FMH Services. Les coûts sont pris en charge par divers sponsors (voir www.fmhservices.ch).

\section{Dates \\ K24 Jeudi \\ K25 Jeudi 15 novembre $2012 \quad$ Genève 17.00-21.30 h Crowne Plaza}

\section{Cabinet de groupe}

Une grande partie des médecins en formation ne peuvent imaginer exercer leur future activité qu'en cabinet de groupe. D'ailleurs, les libres praticiens qui ont un cabinet à la campagne ou même en ville ont de plus en plus de difficultés à trouver un successeur. La création d'un cabinet de groupe ou le regroupement de cabinets existants offre de meilleures chances de succès à long terme et facilite la planification de la relève. Outre les motifs personnels de choix d'un cabinet de groupe, il est indispensable de se pencher au préalable sur les aspects d'économie d'entreprise.

\section{Contenu}

Esprit d'entreprise: Traite des différences entre un cabinet de groupe et un cabinet individuel, et des différentes gestions (entreprise, collaborateurs). Quelles sont les exigences envers le personnel et comment s'organise la planification d'un cabinet de groupe en fonction d'un projet?

Modèles de cabinet / formes juridiques / finances / fiscalité: Cette partie 
sera consacrée à la présentation transparente des différents modèles de cabinets et des formes juridiques possibles (cabinet individuel, système de partenariat junior-senior, infrastructure SA / S.A.R.L., solution SA / S.A.R.L avec intégration globale) et à l'explication des différentes formes de financement du point de vue financier et de la fiscalité. Elle traitera également la question de la gestion et de l'utilisation des moyens financiers et abordera la planification fiscale stratégique et opérationnelle (y. c. la question de la TVA dans les cabinets de groupe).

Assurances / Prévoyance professionnelle: Quelles sont les principales différences en matière de prévoyance entre une personnalité juridique (SA, S.A.R.L) et une entreprise individuelle? Quels sont les différents besoins individuels (par ex. médecin avec ou sans enfants) en matière de couverture d'assurance et de prévoyance professionnelle dans un cabinet de groupe? Quels aspects d'assurance responsabilité civile faut-il prendre en compte (qui doit conclure quoi et quand?) et quelles sont les implica- tions fiscales pour une personne juridique? Qu'en est-il de la prévoyance privée?

Rapport d'expérience: Pour clore le séminaire, un médecin (co-fondateur d'un cabinet de groupe) de la région fait part de ses expériences quant à la création, les difficultés (où, quand et comment un soutien externe est nécessaire), l'organisation et les processus de gestion d'un cabinet de groupe.

\section{Frais/Sponsors}

Gratuit pour les membres FMH Services. Les coûts sont pris en charge par divers sponsors (voir www.fmhservices.ch).

\section{Date}

$\begin{array}{ll}\text { K85 Jeudi } & \text { Lausanne } \\ 20 \text { septembre } 2012 & \text { World } \\ 13.30-18.00 \mathrm{~h} & \text { Trade Center }\end{array}$

10 ans avant le départ à la retraite

Le séminaire s'adresse aux médecins qui ne veulent pas laisser l'organisation de leur retraite (anticipée ou non) au hasard.

\section{Contenu}

Ma rente va-t-elle suffire? Questions relatives au besoin éventuel de capital, aux solutions possibles de prévoyance professionnelle et à une démarche fiscale optimalisée. Fiduciaire et prévoyance vieillesse? Possibilités d'harmoniser une planification idéale du départ à la retraite avec la vente éventuelle du cabinet médical afin de réaliser des économies fiscales importantes. Planification de la prévoyance vieillesse! Estimation précise des besoins futurs sur la base des possibilités de planification existantes et planning optimal des futurs revenus tenant compte du montant, des impôts et de la sécurité.

\section{Coûts}

100 CHF (y compris documents de cours et collations).

\section{Date}

$\begin{array}{lll}\text { K40 Jeudi } & \text { Lausanne } \\ 26 \text { avril } 2012 & \text { World } \\ 17.00-20.00 \mathrm{~h} & \text { Trade Center }\end{array}$

\section{Apertura e rilevamento di uno studio medico}

Il seminario è destinato ai medici in procinto di aprire o di rilevare uno studio medico.

\section{Contenuto}

Business plan (preparazione del piano di finanziamento e del credito d'esercizio, prestito bancario), Pianificazione (insediamento, progetto e pianificazione, scelta del mobilio, budget), Valutazione di uno studio medico (inventario e goodwill), Amministrazione di uno studio medico (interna allo studio, rapporti con la banca), Assicurazioni (tutte le assicurazioni necessarie interne ed esterne allo studio), Passaggio dallo stato di dipendente a quello di indipendente, fiscalità.

\section{Documentazione}

Basato su una check-list per l'apertura di uno studio medico, il raccoglitore, consegnato ad ogni partecipante, contiene tutte le informazioni utili durante e dopo il seminario.

\section{Spese/Sponsor}

Gratuito per membri FMH Services. Diversi sponsor si fanno carico delle spese (si rimanda al sito www.fmhservices.ch).

\section{Date}

\begin{tabular}{|lll|}
\hline K50 & $\begin{array}{l}\text { Martedì } \\
6 \text { marzo } 2012 \\
\text { dalle } 17.00 \text { alle } 21.00\end{array}$ & $\begin{array}{l}\text { Chiasso } \\
\text { FMH Fiduciaria } \\
\text { Services }\end{array}$ \\
\hline K51 & $\begin{array}{l}\text { Mercoledì } \\
16 \text { maggio } 2012 \\
\text { dalle } 14.00 \text { alle } 18.00\end{array}$ & $\begin{array}{l}\text { Chiasso } \\
\text { FMH Fiduciaria }\end{array}$ \\
\hline K52 & $\begin{array}{l}\text { Mervices } \\
\text { 26 settembre } 2012 \\
\text { dalle } 14.00 \text { alle } 18.00\end{array}$ & $\begin{array}{l}\text { Chiasso } \\
\text { FMH Fiduciaria }\end{array}$ \\
\hline K53 & $\begin{array}{l}\text { Martedì } \\
\text { 20 novembre } 2012 \\
\text { dalle } 17.00 \text { alle } 21.00\end{array}$ & $\begin{array}{l}\text { Chiasso } \\
\text { FMH Fiduciaria } \\
\text { Services }\end{array}$ \\
\hline
\end{tabular}

\section{Inscription et information / Iscrizioni e} informazioni

www.fmhservices.ch ou FMH Consulting Services, Cornelia Steinmann, Burghöhe 1, 6208 Oberkirch, Tél. 04192500 77, Fax 041 9210586.

\section{Remarque / Osservazioni}

Les adresses des participants aux séminaires dont les coûts sont couverts en partie ou totalement par des sponsors sont communiquées aux sponsors concernés.

Gli indirizzi dei partecipanti ai seminari, i cui costi sono coperti in parte o completamente da degli sponsor, vengono comunicati agli sponsor interessati.

\section{Conditions d'annulation / \\ Condizioni d'annullamento}

Un montant est perçu pour une absence ou une annulation. Il est de:

Un importo verrà rimborsato in caso di assenza o annullamento. Esso sarà di:

- 50 CHF par personne dans les 15 jours avant le début du séminaire/ per persona entro i 15 giorni prima dell'inizio del seminario;

- 100 CHF par personne dans les 7 jours avant le début du séminaire / per persona entro i 7 giorni prima dell'inizio del seminario. 